\title{
Study of parasitic resistance effects in nanowire and nanoribbon biosensors
}

\author{
loannis Zeimpekis ${ }^{1 *}{ }^{*}$, Kai Sun $^{1+}$, Chunxiao Hu${ }^{1}$, Owain Thomas ${ }^{2}$, Maurits RR de Planque ${ }^{1}$, Harold MH Chong ${ }^{1}$, \\ Hywel Morgan ${ }^{1}$ and Peter Ashburn ${ }^{1}$
}

\begin{abstract}
In this work, we investigate sensor design approaches for eliminating the effects of parasitic resistance in nanowire and nanoribbon biosensors. Measurements of $\mathrm{pH}$ with polysilicon nanoribbon biosensors are used to demonstrate a reduction in sensitivity as the sensor length is reduced. The sensitivity (normalised conductance change) is reduced from $11 \%$ to $5.5 \%$ for a pH change from 9 to 3 as the sensing window length is reduced from 51 to $11 \mu \mathrm{m}$. These results are interpreted using a simple empirical model, which is also used to demonstrate how the sensitivity degradation can be alleviated by a suitable choice of sensor window length. Furthermore, a differential sensor design is proposed that eliminates the detrimental effects of parasitic resistance. Measurements on the differential sensor give a sensitivity of 15\%, which is in good agreement with the predicted maximum sensitivity obtained from modeling.
\end{abstract}

Keywords: Biosensor; Nanowire; Nanoribbon; Parasitic resistance; Differential biosensor; pH sensor

\section{Background}

Over the past 40 years, Ion Sensitive Field Effect Transistors (ISFETs) have been widely researched for applications as ion [1,2], $\mathrm{pH}[3]$, and protein sensors [4]. More recently, nanowire and nanoribbon biosensors $[5,6]$ have been developed as improved devices because their high surface-to-volume ratio gives high sensitivity. Nanowires can be fabricated using bottom-up [7] or top-down [8-13] processes. The bottom-up approach has the advantage of simplicity, but there is no precise control of nanowire size and position, and this makes it difficult to achieve low resistance ohmic contacts. Top-down approaches overcome these shortcomings and can be based around silicon CMOS [14] or thin-film transistor (TFT) [15] technologies. Both approaches are compatible with mass manufacture and allow heavily doped contact regions to be incorporated to reduce contact resistance. However, the cost of a biosensor is determined by the number of steps in the manufacturing process, so the inclusion of heavily doped contacts increases cost.

\footnotetext{
*Correspondence: izk@ecs.soton.ac.uk

${ }^{\dagger}$ Equal contributors

'Zepler Institute, School of Electronics \& Computer Science, University of Southampton, Southampton SO17 1BJ, UK

Full list of author information is available at the end of the article
}

The sensitivity of a biosensor is defined as the relative change in conductance after attachment of the target biomolecule and is inversely proportional to the doping concentration in the nanowire or nanoribbon [16]. Consequently, biosensors that are designed for high sensitivity have a relatively large resistivity, and high values of contact resistance are likely unless additional heavily doped contact regions are incorporated into the manufacturing process. Moreover, to accommodate measurements in a liquid environment, biosensor metallization and contact regions are covered with a passivation layer to protect them from the analyte solution. The passivated regions are not influenced by the attachment of target biomolecules to the surface of the sensor and therefore act as a parasitic resistance. When protein sensing is performed, the relative current changes can be rather small for some proteins [15]. In this situation, signal read-out considerations require sensor operation at higher currents, where the effects of parasitic resistance can be important. To date, there have been no published studies of the effect of parasitic resistance on the performance of nanowire or nanoribbon biosensors.

The aim of this work is to investigate the effects of parasitic resistance on biosensor sensitivity. Thin-film polysilicon nanoribbon biosensors are fabricated with 
different sensing window lengths, and it is shown that the sensitivity is lower for shorter sensing windows. These results are interpreted using a simple empirical model that relates biosensor sensitivity to parasitic resistance and sensing window length. Finally, a differential biosensor design is demonstrated that eliminates the detrimental effects of parasitic resistance and delivers a value of sensitivity equal to the maximum predicted by the model. This high value of sensitivity is achieved without the need to incorporate heavily doped contact regions.

\section{Methods}

Polysilicon nanoribbon biosensors were fabricated with different channel lengths using the TFT process detailed in our previous work [6] and measured in dry and wet ambient. Electrical characterization was performed using an Agilent B1500A I/V-based probestation (Agilent Technologies Singapore (International) Pte. Ltd., Singapore). The sensors were measured in a Faraday cage enclosure box to minimize interference. For the characterization of the sensors, electrical contact was made on the TiN electrodes using Cascade micropositioner probes. The backside of the substrate was grounded through the probe-station chuck to prevent biasing of the channel through it. Transmission line measurements (TLMs) were performed on test structures, and values of sheet resistance $\left(R_{\mathrm{s}, \mathrm{dry}}\right)$ and contact resistance $\left(R_{\mathrm{c}}\right)$ were extracted to be $448 \mathrm{k} \Omega / \mathrm{sq}$ and $447 \mathrm{k} \Omega$, respectively. From these values, the total parasitic resistance $\left(R_{\text {par }}\right)$ was calculated to be $1.6 \mathrm{M} \Omega$.
For wet sensing with $\mathrm{pH}$ buffers, a liquid gate was formed by pipetting liquid in the sensing window while a potential was applied using a $\mathrm{Ag} / \mathrm{AgCl}$ electrode immersed in the solution. $\mathrm{pH}$ measurements were carried out using universal buffer mixture (UBM) solutions of $\mathrm{pH} 3,5,7$, and 9. Those measurements were initiated by introducing $\mathrm{pH} 9$ in the sensor window while applying a $50 \mathrm{mV}$ bias to a $\mathrm{Ag} / \mathrm{AgCl}$ electrode and a channel potential of $100 \mathrm{mV}$. After a $10 \mathrm{~min}$ stabilization time, the measurement was reset and the buffers were introduced sequentially from high to low $\mathrm{pH}$ and back to high $\mathrm{pH}$ to complete a full titration. All sensing measurements were performed at the same sensor biasing point so that values of sensitivity could be quantitatively compared.

\section{Results and discussion}

Figure 1 shows the normalized conductance change as a function of time when the biosensor is exposed to different $\mathrm{pH}$ buffers for two different TFT biosensors with sensing window lengths of 51 and $11 \mu \mathrm{m}$. For the $51 \mu \mathrm{m}$ window sensor, the conductance increases by approximately $11 \%$ for a $\mathrm{pH}$ change of 6 units, from $\mathrm{pH} 9$ to $\mathrm{pH}$ 3. A subsequent increase in $\mathrm{pH}$ results in a decrease of conductance back to the original value. A similar, though smaller, trend is observed for the sensor with the shorter sensing window of $11 \mu \mathrm{m}$ with a conductance increase of $5.5 \%$ from $\mathrm{pH} 9$ to $\mathrm{pH}$ 3. Therefore, the longer $51 \mu \mathrm{m}$ sensor has a higher sensitivity than the shorter $11 \mu \mathrm{m}$ sensor. This indicates a relation between sensitivity and sensing window length.

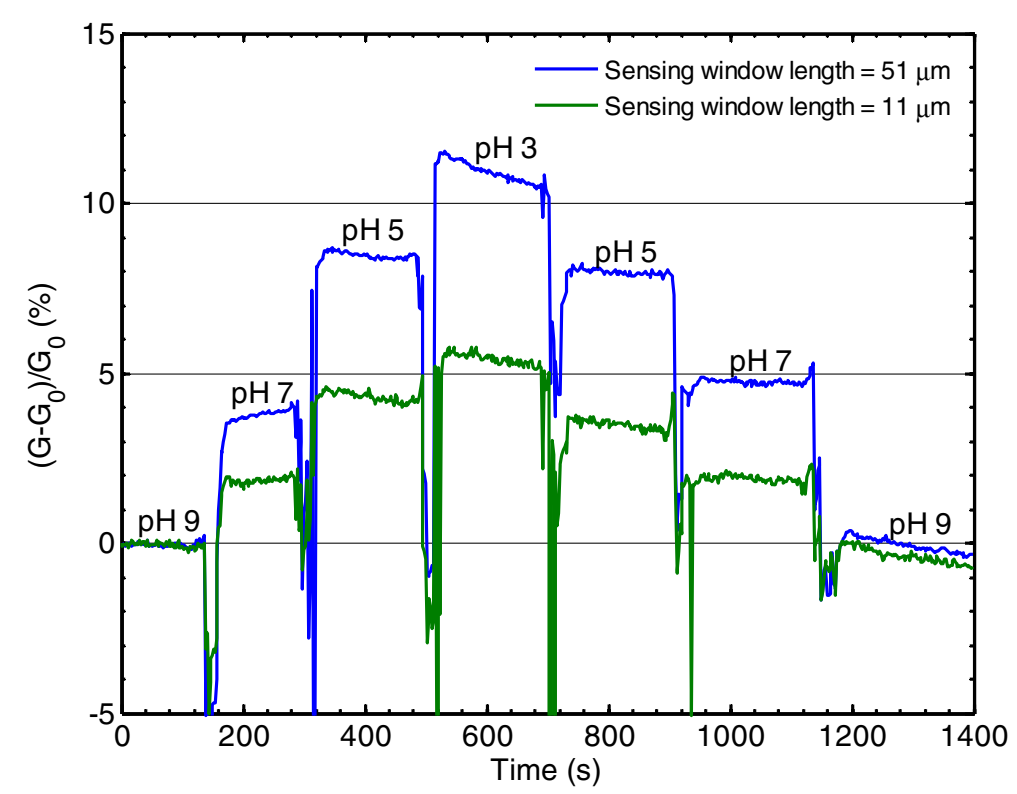

Figure 1 Normalized conductance change (sensitivity) measured after changes of $\mathrm{pH}$ for two sensors with different sensing window lengths. 
To study the relationship between sensitivity and sensor dimensions, a simple model is presented in Figure 2. The model includes three sections; the wet section $\left(R_{\text {sense }}\right)$ where the sensor is exposed to liquid and two dry sections $\left(R_{\text {par }}\right)$ where it is protected from liquid by SU8. All sections contribute to the overall resistance $(R)$, which can be measured directly from electrical measurements. The dry section includes the contact resistance from the TiN/Si contacts $\left(R_{\mathrm{c}}\right)$ and the parasitic resistance of the nanoribbon region covered by SU8 $\left(R_{\text {rbd }}\right)$.

The total resistance of the biosensor is:

$$
\begin{aligned}
R & =R_{\text {sense }}+R_{\text {par }}=R_{\text {sense }}+2 R_{\text {rbd }}+2 R_{\mathrm{c}} \\
& =\frac{R_{\mathrm{s}, \text { wet }} L_{\mathrm{s}}}{W_{\mathrm{rb}}}+2 \frac{R_{\mathrm{s}, \mathrm{dry}} L_{\mathrm{d}}}{W_{\mathrm{rb}}}+2 R_{\mathrm{c}}
\end{aligned}
$$

where $R_{\mathrm{s}}$,wet and $R_{\mathrm{s} \text {,dry }}$ are the sheet resistances of the wet and dry nanoribbon regions, $L_{\mathrm{s}}$ and $L_{\mathrm{d}}$ are the lengths of the wet and dry nanoribbon regions, $W_{\mathrm{rb}}$ is the nanoribbon width, and $R_{\mathrm{c}}$ is the contact resistance of the TiN/Si contacts. Here, $R_{\mathrm{s}, \mathrm{dry}}$ and $R_{\mathrm{c}}$ can be measured using TLM test structures. The dimensions of the device, $L_{\mathrm{s}}, L_{\mathrm{d}}$, and $W_{\mathrm{rb}}$, can be measured optically. Using (1), $R_{\mathrm{s} \text {,wet }}$ can be calculated from the measured biosensor resistance, $R$. The sensitivity is defined as the normalized conductance change and is given by:

$$
\text { Sensitivity }=\frac{G-G_{0}}{G_{0}}=\frac{1 / R-1 / R_{0}}{1 / R_{0}}
$$

where $R_{0}$ and $G_{0}$ are the resistance and the conductance at a reference level (pH 9 in this work), respectively.

Figure 3 shows the modeled sensitivity as a function of sensing window length (solid lines) calculated with parameters obtained from measurements on a sensor with a sensing window length of $51 \mu \mathrm{m}$. The measured sensitivities of two devices with sensing window lengths of 11 and $31 \mu \mathrm{m}$ are also presented (points). The curves closely match the measured data indicating that the experimental data can be modeled with reasonable accuracy.
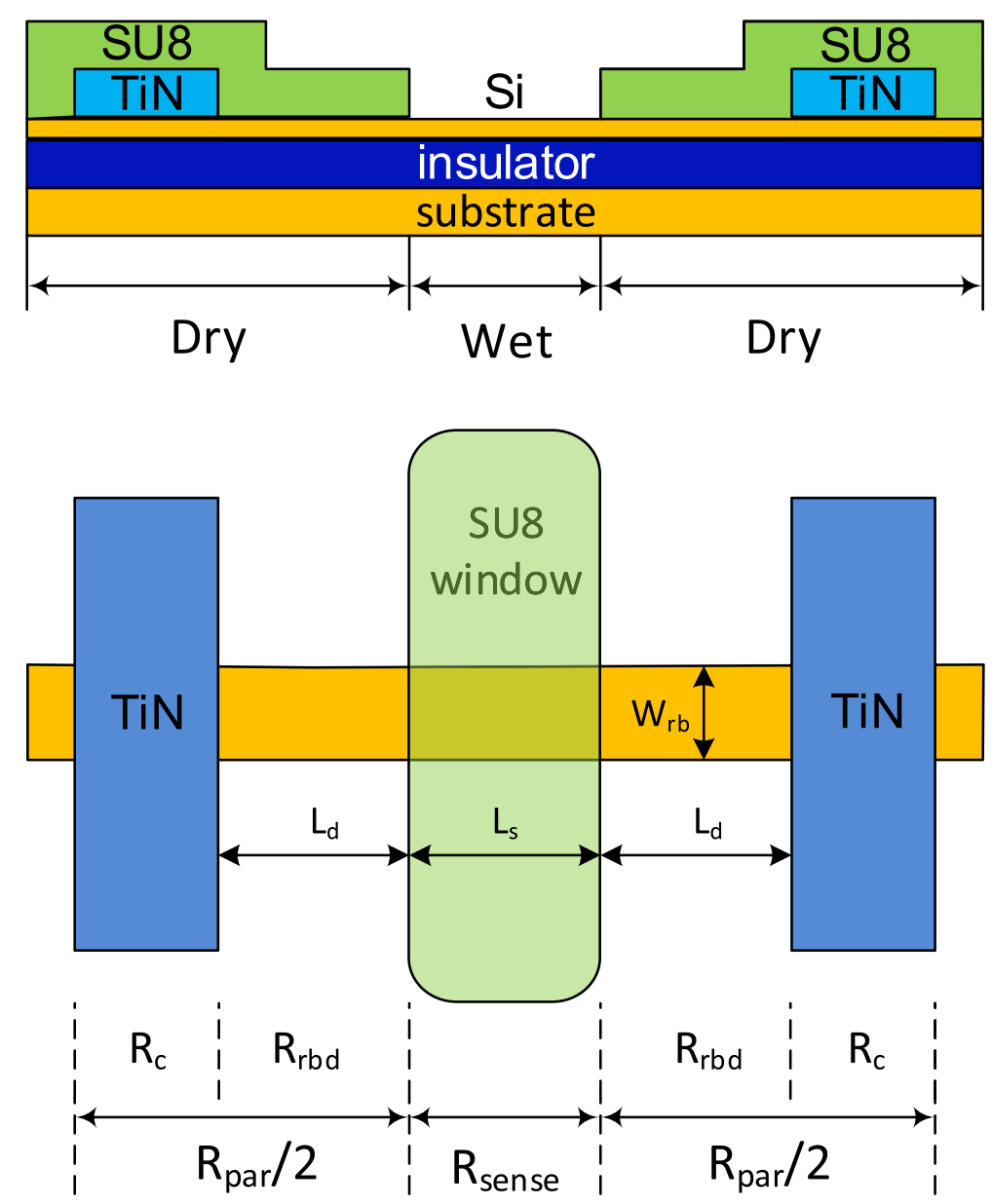

Figure 2 Schematic cross-section and plan views of the polysilicon nanoribbon biosensor. 


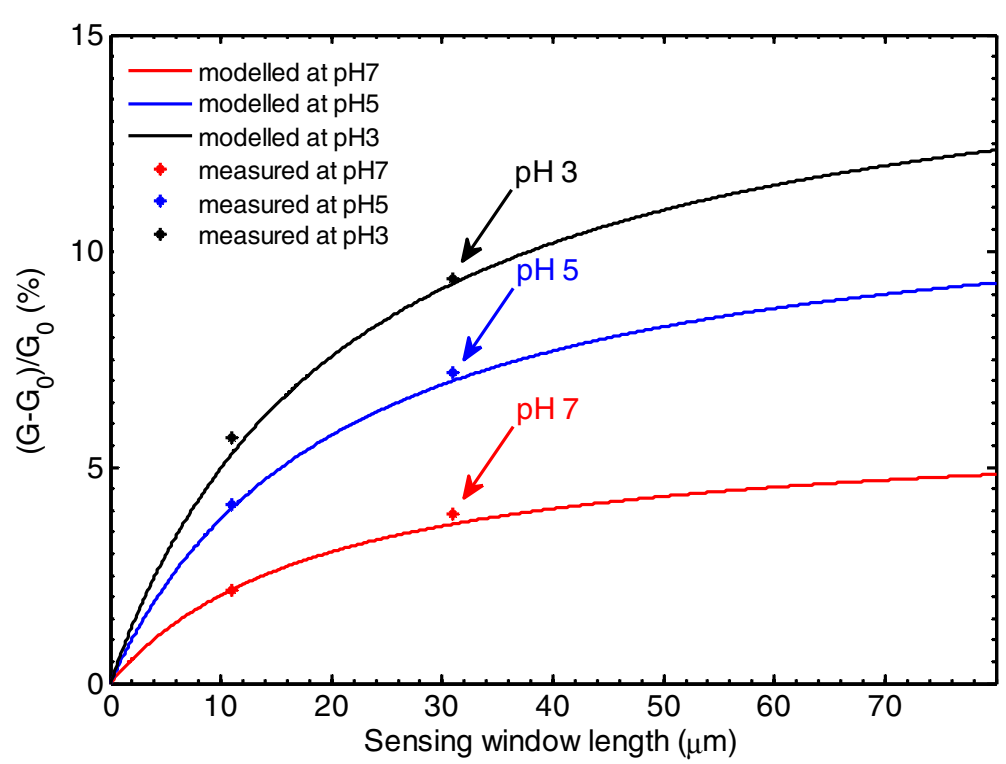

Figure 3 Modeled normalized conductance change (sensitivity) as a function of sensing window length at different values of pH. The graph also includes measured data from two devices with sensing window lengths of 11 and $31 \mu \mathrm{m}$.

To investigate how practical sensors can be designed for maximum sensitivity, the normalized conductance change at $\mathrm{pH} 3$ is plotted in Figure 4 over a wide range of sensing window lengths. The green line in Figure 4 is plotted using measured data from the fabricated sensors and used a parasitic resistance of $1.6 \mathrm{M} \Omega$. For comparison, the red and blue lines show predictions for different values of parasitic resistance of $800 \mathrm{k} \Omega$ and $3.2 \mathrm{M} \Omega$, respectively. For an ideal sensor, with a parasitic resistance of zero, the sensitivity remains constant with a value of $15.6 \%$. In contrast, for the three sensors with parasitic resistance, the sensitivity approaches the ideal value at long channel lengths. In order to achieve a sensitivity of $90 \%$ of the maximum (14.1\%), biosensor lengths of 85 , 185 , or $380 \mu \mathrm{m}$ are required for values of parasitic resistance of $800 \mathrm{k} \Omega, 1.6 \mathrm{M} \Omega$, and $3.2 \mathrm{M} \Omega$, respectively. This result shows that to approach the maximum value of sensitivity, biosensors with long lengths must be used.

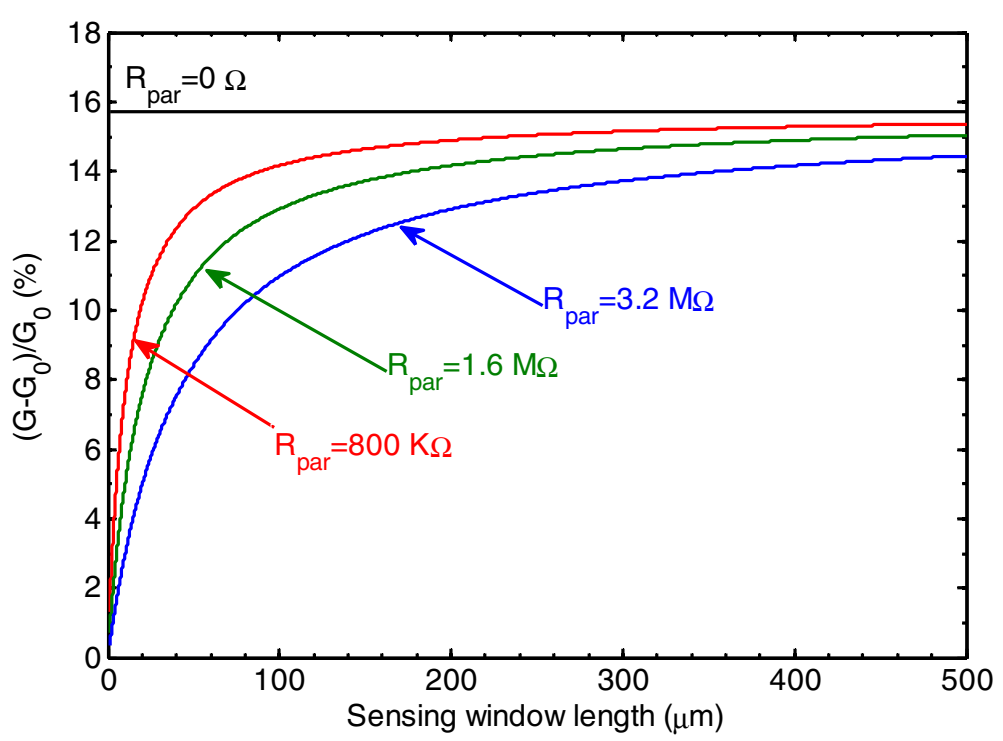

Figure 4 Predicted normalized conductance change as a function of window length for different values of parasitic resistance. 


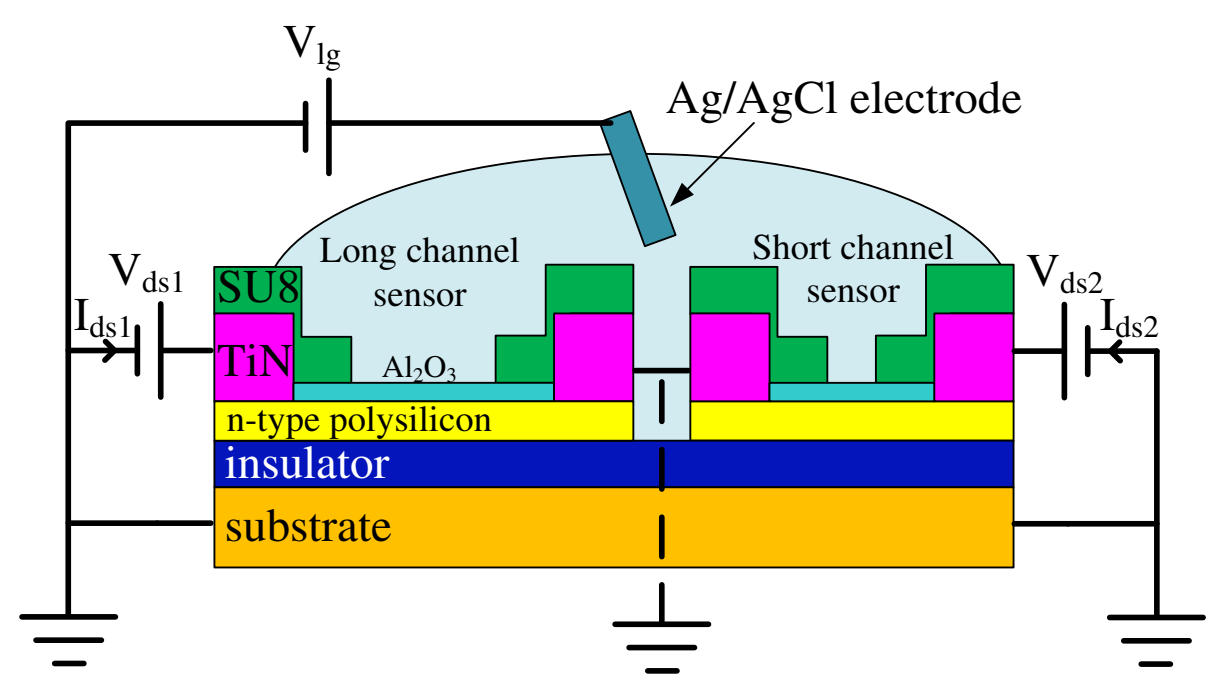

Figure 5 Schematic illustration of the differential design and measurement configuration. Two sensors are used for the differential measurement, with long and short sensing windows. The sensors are otherwise identical.

This conclusion contradicts the model presented in [16], which predicted that the biosensor sensitivity increased for shorter channel lengths. However, the model presented in [16] did not include the effects of parasitic resistance and hence predicts the behavior of idealised, rather than practical, biosensors.

To maximize the sensitivity, a differential biosensor design is proposed in Figure 5, which measures the difference in resistance between the two biosensors with different sensor window lengths. As the two biosensors have equal values of parasitic resistance $\left(R_{\mathrm{par} 1}=R_{\mathrm{par} 2}\right)$ but different window lengths $\left(R_{\text {sense1 }} \neq R_{\text {sense } 2}\right)$, subtraction eliminates the parasitic resistance from the equation. This differential sensor design therefore should eliminate the parasitic resistance effects and give the maximum value of sensitivity.

Figure 6 shows the measured normalized conductance change as a function of sensor window length for the differential sensor. The conductance change follows a similar trend to the results in Figure 1 for the individual sensors, but the change is larger. The normalized conductance change between $\mathrm{pH} 9$ and $\mathrm{pH} 3$ is $15 \%$. This is

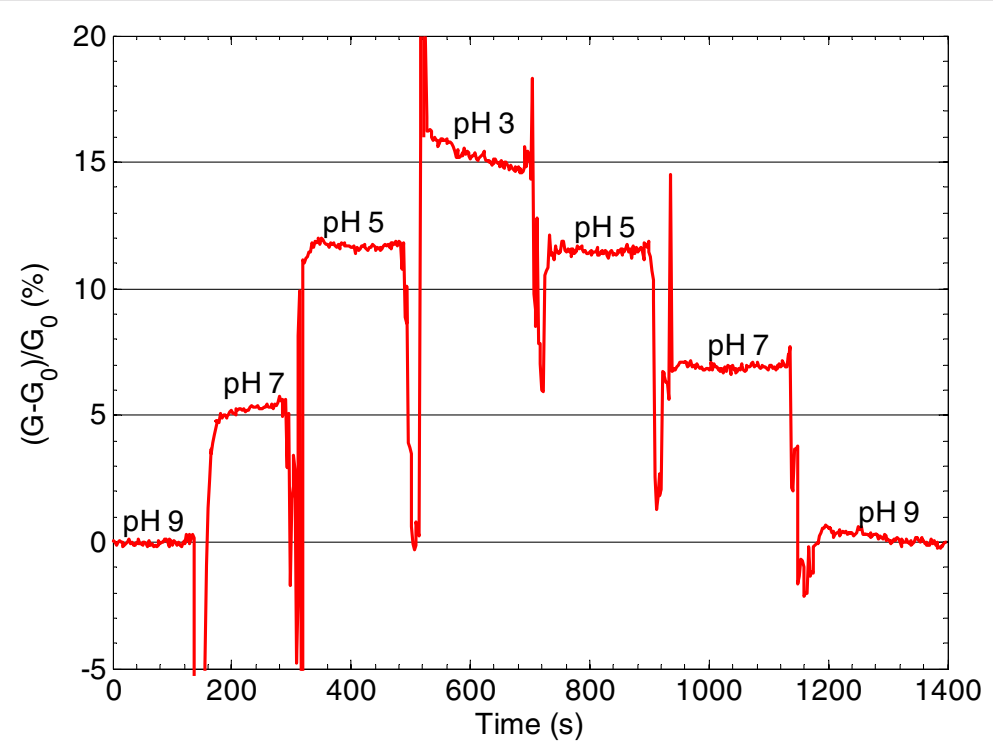

Figure 6 Measured normalized differential conductance change (sensitivity) for the differential sensor after changes in solution pH. 
Table 1 Comparison of measured sensitivity of the differential biosensor with modeled maximum values (for parasitic resistance equal to zero)

\begin{tabular}{lll}
\hline pH value & $\begin{array}{l}\text { Differentially measured } \\
\text { sensitivity }\end{array}$ & $\begin{array}{l}\text { Predicted maximum } \\
\text { sensitivity }\end{array}$ \\
\hline 7 & $6.0 \%$ & $6.0 \%$ \\
5 & $11.5 \%$ & $11.6 \%$ \\
3 & $15.1 \%$ & $15.6 \%$ \\
\hline
\end{tabular}

a $37 \%$ improvement over the result from the $51-\mu \mathrm{m}$ long sensor and a $174 \%$ over the $11 \mu \mathrm{m}$ sensor.

Table 1 compares the measured values of sensitivity obtained using the differential sensor design with predicted maximum values of sensitivity obtained from the model results in Figure 3. The differentially measured values of sensitivity are in excellent agreement with the predicted maximum values of sensitivity for all $\mathrm{pH}$ values. It can consequently be concluded that the differential sensor design presented here has eliminated the detrimental effects of parasitic resistance and delivered the maximum value of sensitivity that is possible for a given nanoribbon doping concentration.

The sensing window lengths of the devices measured in this work were chosen so that the performance of the individual sensors in the differential pair could be separately demonstrated. The requirement for the differential sensor to work is that both sensors have the same parasitic resistance but different sensing window lengths. In practice, a smaller differential biosensor footprint could be achieved if the sensing window length of one sensor was set to zero. When performing the differential measurement, the zero length sensor would then cancel the parasitic resistance of the finite length sensor. This approach allows the differential sensor design to be miniaturized, while maintaining the maximum sensitivity.

\section{Conclusions}

This paper has studied the effect of parasitic resistance on biosensor sensitivity. The results show that a high value of parasitic resistance reduces the sensitivity of the sensor, while a long sensing window reduces this sensitivity degradation. A model has been formulated to describe this relationship and used to identify how the sensing window length can be chosen to minimize the negative effects of parasitic resistance. Furthermore, a differential biosensor design has been proposed, and the measurements have indicated that the deleterious effects of parasitic resistance have been eliminated.

\section{Competing interests}

The authors declare that they have no competing interests.

\section{Authors' contributions}

IZ and KS conceived the idea and worked equally for this study. They designed, fabricated and tested the sensors, formulated the model, and drafted the manuscript. $\mathrm{CH}$ prepared all necessary solutions and assisted with the measurements. OT advised and assisted on the fabrication of the devices. MRRDP and HMHC advised the authors throughout the study. HM and PA participated on the design, coordinated this study and helped to draft the manuscript. All authors read and approved the final manuscript.

\section{Acknowledgement}

The authors would like to acknowledge the Technology Strategy Board (TSB) and the Engineering and Physical Sciences Research Council (EPSRC: EP/K502327/1) for funding this work. We would also like to thank Ben Hadwen, Sally Anderson, Gregory Gay and Chris J. Brown of Sharp Laboratories Europe for many useful discussions.

\section{Author details}

${ }^{1}$ Zepler Institute, School of Electronics \& Computer Science, University of Southampton, Southampton SO17 1BJ, UK. ${ }^{2}$ Oxford Instruments Plasma Technology, Yatton, Bristol BS49 4AP, UK.

Received: 24 November 2014 Accepted: 30 January 2015

Published online: 21 February 2015

\section{References}

1. Bergveld P. Thirty years of ISFETOLOGY: What happened in the past 30 years and what may happen in the next 30 years. Sensors Actuators $B$ Chem. 2003:88:1-20.

2. Chen Y, Xihua W, Erramilli S, Mohanty P, Kalinowski A. Silicon-based nanoelectronic field-effect pH sensor with local gate control. Appl Phys Lett. 2006:89:223512.

3. Bausells J, Carrabina J, Errachid A, Merlos A. Ion-sensitive field-effect transistors fabricated in a commercial CMOS technology. Sensors Actuators B Chem. 1999;57:56-62.

4. Zheng GF, Patolsky F, Cui Y, Wang WU, Lieber CM. Multiplexed electrical detection of cancer markers with nanowire sensor. Nat Biotechnol. 2005;23:1294-301.

5. Curreli M, Rui Z, Ishikawa FN, Chang HK, Cote RJ, Chongwu Z, et al. Real-time, label-free detection of biological entities using nanowire-based FETs. IEEE Trans Nanotechnol. 2008;7:651-67.

6. Sun K, Zeimpekis I, Lombardini M, Ditshego NMJ, Pearce SJ, Kiang KS, et al. Three-mask polysilicon thin-film transistor biosensor. IEEE Trans Electron Devices. 2014;61:2170-6.

7. Chui Y, Wei Q, Park H, Lieber CM. Nanowire nanosensors for highly sensitive and selective detection of biological and chemical species. Science. 2001:17:1289-92.

8. Li Z, Chen Y, Li X, Kamins TI, Nauka K, Williams RS. Sequence-specific label-free DNA sensors based on silicon nanowires. Nano Lett. 2004:4:245-7.

9. Bunimovich YL, Shin YS, Yeo WS, Amori M, Kwong G, Heath JR. Quantitative real-time measurements of DNA hybridization with alkylated nonoxidized silicon nanowires in electrolyte solution. J Am Chem Soc. 2006;128:16323-31.

10. Dorvel BR, Reddy Jr B, Go J, Guevara CD, Salm E, Alam MA, et al. Silicon nanowires with high-k hafnium oxide dielectrics for sensitive detection of small nucleic acid oligomers. ACS Nano. 2012;6:6150-64.

11. Park IY, Li ZY, Li XM, Pisano AP, Williams RS. Towards the silicon nanowirebased sensor for intracellular biochemical detection. Biosens Bioelectron. 2007:22:2065-70.

12. Tarasov A, Wipf M, Bedner K, Kurz J, Fu W, Guzenko VA, et al. True reference nanosensor realized with silicon nanowires. Langmuir. 2012;28:9899-905

13. Zhang GJ, Zhang L, Huang MJ, Luo ZHH, Tay GKI, Lim EJA, et al. Silicon nanowire biosensor for highly sensitive and rapid detection of Dengue virus. Sensors Actuators B Chem. 2010;146:138-44.

14. Stern E, Klemic JF, Routenberg DA, Wyrembak PN, Turner-Evans DB, Hamilton AD, et al. Label-free immunodetection with CMOS-compatible semiconducting nanowires. Nature. 2007:445:519-22.

15. Hakim MMA, Lombardini M, Sun K, Giustiniano F, Roach PL, Davies DE, et al. Thin film polycrystalline silicon nanowire biosensors. ACS Nano Lett. 2012;12:1868-72.

16. Nair PR, Alam MA. Design considerations of silicon nanowire biosensors. IEEE Trans Electron Devices. 2007;54:3400-8. 